\title{
EVALUATION OF 2-METHACRYLOYLOXYETHYL PHOSPHORYLCHOLINE POLYMER COATED POLYAMIDE DENTURE BASE MATERIAL: SURFACE ROUGHNESS AND CANDIDA ALBICANS ADHESION
}

\author{
Tayseer Maaly* and Eman Husseiny Mohamed ${ }^{* *}$
}

\begin{abstract}
Purpose: To evaluate surface roughness and Candida albicans adhesion of polyamide resin denture base coated with various concentrations of MPC polymer, 2-methacryloyloxyethyl phosphorylcholine.

Materials and Methods: A total of eighty polyamide disks were prepared and divided into four groups ( $n=20$ /group). Surfaces of specimens in three groups were treated with MPC in various concentrations $(0.25 \mathrm{~mol} / \mathrm{L} ; 0.50 \mathrm{~mol} / \mathrm{L}$ and $0.75 \mathrm{~mol} / \mathrm{L})$, whereas uncoated specimens were used as control. Surface roughness (SR) was evaluated using a profilometer. Incubation of the specimens with $C$. albicans was done overnight, and the count of adhered cells was estimated. Statistically, the following tests were done: analysis of variance, Pearson correlation coefficient test, post hoc Tukey test.
\end{abstract}

Results: Upon PMPC coating, surface roughness values were decreased, with a significant difference between the groups. There was significant decrease in $C$. albicans count with the increase in the MPC polymer concentration. For the modified groups, $0.50 \mathrm{~mol} / \mathrm{L}$ and $0.75 \mathrm{~mol} / \mathrm{L}$, showed no significant difference for candida adhesion while significantly different for surface roughness. These results showed that polymerization of MPC by graft method on polyamide denture base material decreases the attachment of $C$.albicans, consequently, it is recommended as a coating for elimination of denture stomatitis.

Conclusion: modification of polyamide prosthesis with $0.75 \mathrm{~mol} / \mathrm{L} \mathrm{MPC}$ is suitable to diminish C. albicans adhesion on the denture base and a decreasing effect on the surface roughness values.

KEYWORDS: Candida adhesion, Polyamide resin, MPC, Surface roughness

\footnotetext{
* Lecturer, Dental Biomaterials Department, Faculty of Dentistry, Zagazig University.

** Dental Prosthodontics Department, Faculty of Dentistry, Zagazig University
} 


\section{INTRODUCTION}

Polymethyl methacrylate (PMMA) has been the greatest outstanding material utilized for denture construction next its presentation in 1937. In any case, it has a few issues, for example polymerization contraction, low flexural and impact strength, also reduced fatigue resistance that led to failure of the denture throughout chewing process or once it fall out from hands of the patient. ${ }^{1}$ Besides PMMA denture base materials can possibly cause unfavourable reactions due to monomer release and biofilm-associated infections., ${ }^{2,3}$ As the dentures remain in contact with great areas of the oral mucosa, probability for presence of candida infections and adversely susceptible responses.,5 Alongside the advancement of dentistry science, showed numerous studies to enhance the mechanical properties of acrylic resin.

Throughout the 1950s presented polyamide or thermoplastic resin as a novel denture base material. There is an increase in the use of thermoplastic resins, particularly in the nylon material. ${ }^{6,7}$ Thermoplastic nylon has numerous advantages, which was notable aesthetic, non-toxic properties, increased flexibility, secure to use in patients who have allergy to metals and monomer resin, in addition to its sufficient strength so be used as denture base material. ${ }^{6}$ In spite of these advantages, thermoplastic nylon has problems like increased water permeable, liability to colour changing, difficult manipulation, and increasing surface roughness next few weeks' usage. $^{7}$

Surface roughness is one of denture base material properties which show direct relationship between plaque accumulation and bond of Candida albicans. Studies showed that the surface roughness of thermoplastic nylon resin was three times more than polymethyl methacrylate acrylic resin. ${ }^{4}{ }^{8}$ Chronic atrophic (erythematous) candidiasis, similarly known as denture sore mouth and denture stomatitis, is characterized by confined chronic erythema and oedema of the mucosa which contact the denture fitting surface. Its principal aetiology is the chance for the overgrowth of Candida in the zone between the palate and the surface of denture where natural salivary flow is limited. ${ }^{9,10}$ Consequently, inhibition of adhesion and growth of microorganisms is an effective way for suppression of the development and progress of denture plaque.

2-methacryloyloxyethyl phosphorylcholine (MPC) is an extensively applied biocompatible and hydrophilic biomedical polymer. ${ }^{11,}{ }^{12}$ In 1990 , following the outline of the synthesis and purification process of MPC by Ishihara et $\mathrm{a}^{13}$ in a superior and complete method, significant improvement has been made in the advance of the MPC polymer (PMPC) as a biomaterial. It has been proven to have extensive antithrombogenicity due to the reality of its excellent resistance to cell adhesion and its response to endogenous proteins and cells. ${ }^{14,15}$ -

It was reported that coating of medical devices with PMPC be very useful in decreasing the count of adherent bacteria. ${ }^{11}$ In spite of the use of MPC polymer in medical fields, there is no utilization of MPC in dental applications. There are limited dental studies, ${ }^{11,16,17}$ involving MPC, extraordinarily little studies regarding adhesion of $\mathrm{C}$. albicans on PMPC coated polyamide denture base materials found through the literature studies. Depending on the medical research which have shown MPC polymer to decrease microorganism and cell adhesion, present study was considered to assess PMPC coating with different concentrations onto polyamide denture base material and evaluate the modifications on surface roughness, in addition to C. albicans adhesion which is a frequent factor for denture stomatitis.

\section{MATERIALS AND METHODS}

The calculated sample size of this study with (power $80 \%, 5 \%$ level of significance) was 10 specimens for each test group (total of 80 specimens), 
using G Power 3 sample size calculator. The sample size was increased to 12 specimens for each group to compensate for incomplete data to increase the study power.

\section{Preparation of polyamide resin specimens}

A modelling wax, brand (Berrwax Modelling Wax, Berr Dental, Turkey) was used in this study, eighty thermoplastic polyamide disks (Unbreakable Nylon thermoplastic resin, TCS, INC, USA) measuring (3.0 $\mathrm{mm}$ thickness and $10 \mathrm{~mm}$ diameter), were conventionally prepared according to manufacturer's instructions. Highly polished Stainless-steel molds were used for the fabrication of resin specimens following the manufactures' instructions. To standardize the surfaces, reduction of both aspects of each specimen was performed by means of green mounted stones to reach $2 \mathrm{~mm}$ thickness of each specimen Figure 1.

\section{Modification of the polyamide specimens with MPC}

Preparation of specimens: Ultrasonic cleaning of the surfaces of all specimens was done using both hexane and ethanol for $5 \mathrm{~min}$ first and then followed by $30 \mathrm{~min}$, after cleaning, the specimens were left air dried at room temperature. After that, polyamide specimens were separated in a random manner into four groups. Twenty specimens were haphazardly selected and used as control group as they didn't subject to any surface modification. The other specimens which selected for MPC modification were soaked in a solution of both ethanol, and $10 \mathrm{mg} / \mathrm{mL}$ benzophenone, Sigma-Aldrich, Saint Louis, USA, for a duration of $30 \mathrm{~s}$. Specimens were removed, to remove ethanol, drying of the specimens was performed at room temperature in the dark. ${ }^{11}$

Preparation of coating material: MPC polymer, the material that was used for coating, was purchased from Sigma-Aldrich, Saint Louis, USA. The material was liquified in pure water so attaining the monomer in an aqueous solution at the concentrations: $0.25 \mathrm{~mol} / \mathrm{L}, 0.5 \mathrm{~mol} / \mathrm{L}$ and $0.75 \mathrm{~mol} / \mathrm{L}$.

Coating of the specimens: The prepared specimens that were treated with benzophenone, soaked in the modified solutions of MPC of the different concentrations. A device of ultraviolet ray (White/Ultraviolet Transilluminator TLW-20, UVP, CA, USA) with a wavelength of $365 \mathrm{~nm}$ and power

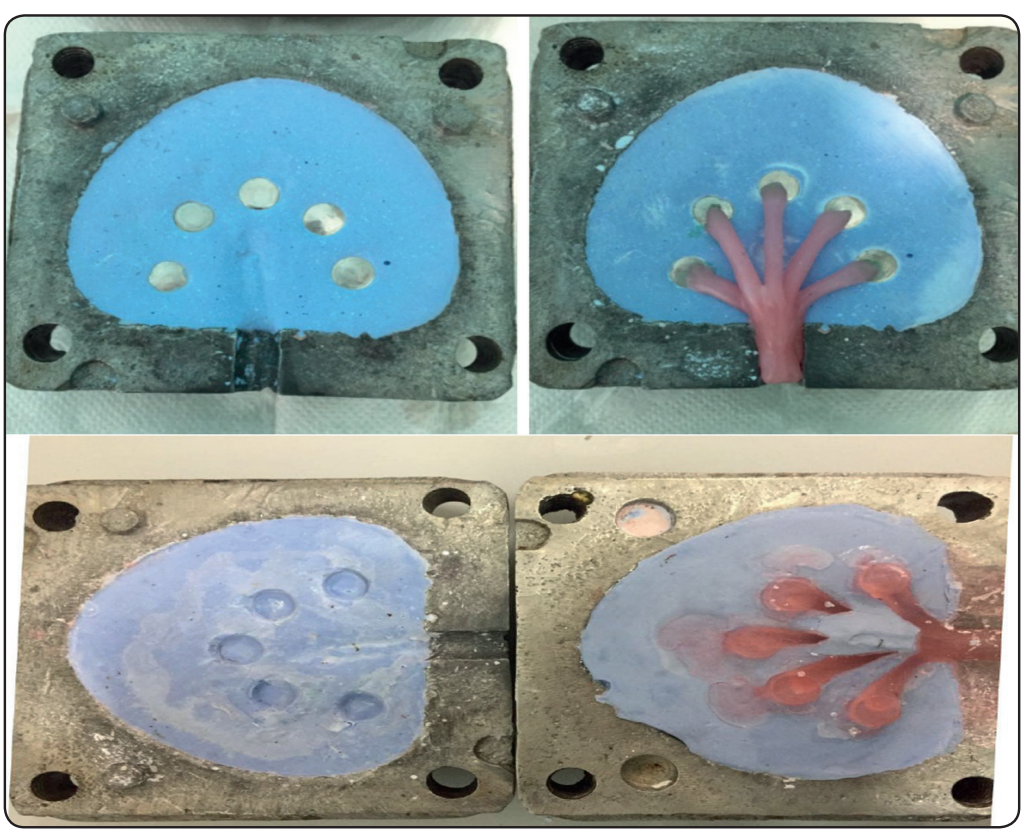

Fig. (1) Preparation of polyamide resin specimens using stainless-steel mold. 
of 8 watts was used to photo-polymerize the coating material, MPC on polyamide specimens. The graft polymerization was performed at $70^{\circ} \mathrm{C}$ for two hours, using the ultraviolet device positioned $20 \mathrm{~cm}$ away from the specimens. After achievement of graft polymerization, PMPC-modified polyamide specimens were washed using distilled water to eliminate remaining monomers, and they were dehydrated at room temperature. The modified specimens were ready for the evaluation of both roughness and candida adhesion and compared with the unmodified ones. ${ }^{11}$

\section{Surface Roughness Test}

The average surface roughness ( $\mathrm{Ra}$ ) of each group was examined using a profilometer (Surftest SJ210, Mitutoyo Corp., Kawasaki, Japan) according to the ISO 4287-1997. For this purpose, specimens were measured of each group and the probe was placed in the middle of the specimen surface. Three values for each specimen were recorded (one reading was recorded at the middle of the specimen, and the other two were recorded at the margins) for whole specimens of each group, and $(\mathrm{Ra})$, mean roughness parameter was estimated in $(\mu \mathrm{m}){ }^{8}$

\section{C. albicans adherence test}

Resin specimens were immersed in $70 \%$ ethanol for $12 \mathrm{~min}$, then they were cleaned in distilled water using ultrasonic device for $5 \mathrm{~min}$ to remove any residues attached to the surface. C. albicans ATCC 10231 provided from department of Microbiology, Faculty of Science, Zagazig University, its concentration was prepared to the level $1.5 \times 10^{8} \mathrm{cfu} /$ $\mathrm{mL}$, in Sabourraud dextrose broth, SDA (Acumedica Co., Manufacturers, Inc.) medium. Specimens were placed in $2 \mathrm{~mL}$ of Sabouraud dextrose broth medium in sterilized tubes containing $100 \mu \mathrm{L} C$. albicans suspension, then incubated at $37^{\circ} \mathrm{C}$ for $18 \mathrm{~h}$ overnight. After incubation, removal of specimens and cleaning using phosphate buffered saline (PBS) for three times, then each one was sited into $1 \mathrm{~mL}$ sterile PBS placed in Eppendorf tubes, and followed by vortexed vibration for $2 \mathrm{~min}$ to separate the attached yeast cells. They were immersed in SDA medium and incubated at $37^{\circ} \mathrm{C}$ for $24 \mathrm{~h}$. C. albicans adherend to the specimens was counted, determination of Candida colonies was done one time per specimen and adherent cells number was specified as colony forming units $(\mathrm{CFU}) / \mathrm{mL} .^{5}$

\section{Statistical Analysis}

Data was analysed by means of Statistical Package for Social Science software computer system version 23 (SPSS, Inc., Chicago, IL, USA). Mean and standard deviation values were estimated. For the comparison between two different groups, Student's t-test was applied, while for the comparison between more than two different groups, (ANOVA) one-way analysis of variance, and post-hoc Tuckey test was applied. A statistically significant results when the $P$ value recorded less than 0.05 . Pearson's correlation coefficient was used to correlate both the surface roughness \& Candida adhesion test. When $\mathrm{P}$ value was lesser than 0.05 , the corelation was statistically significant.

\section{RESULTS}

Means and standard deviations of roughness values of evaluated groups are shown in Figure 2. ANOVA analysis for surface roughness values determined a significant difference among the groups. In comparison to the control group, by the increase in the ratios of grafted MPC to the polyamide specimens, there was a significant decrease in surface roughness. The lowest roughness value was recorded for MPC concentration of $0.75 \mathrm{~mol} / \mathrm{L}$ $(0.55 \pm 0.12)$, which showed significantly decreased roughness value in a comparison to the unmodified and modified tested groups, $(\mathrm{p}<0.001)$. While the highest values were recorded for the unmodified group $(0.96 \pm 0.16)$. Among the modified groups, $0.25 \mathrm{~mol} / \mathrm{L} \mathrm{MPC}$ and $0.5 \mathrm{~mol} / \mathrm{L} \mathrm{MPC}$ showed no significant difference $(\mathrm{P}>0.05)$ with the lowest value for $0.5 \mathrm{~mol} / \mathrm{L} \mathrm{MPC}$, and both groups showed a significant decrease in Ra in comparison to control group $(\mathrm{P}<0.05)$. 
Figure 3 showed means and standard deviations combined with the statistical significances of $\mathrm{C}$. albicans adhesion for the different groups. ANOVA analysis for candida count presented significant differences among the groups. The lowest Candida count was recorded for $0.75 \mathrm{~mol} / \mathrm{LMPC}$ followed by $0.5 \mathrm{~mol} / \mathrm{L} \mathrm{MPC} \mathrm{(} 857 \pm 539.7$ and $968 \pm 635.3$ ) without significant difference between the two modified groups $(\mathrm{P}>0.05)$, While the highest adhesion was recorded for the control group followed by 0.25 $\mathrm{mol} / \mathrm{L} \mathrm{MPC}(8163 \pm 3.81$ and $4796 \pm 2811)$ showing a difference which is statistically significant $(\mathrm{P}<0.05)$. Pearson correlation coefficient test is shown in Figure 4, revealed a significant positive correlation between SR and bacterial adhesion.

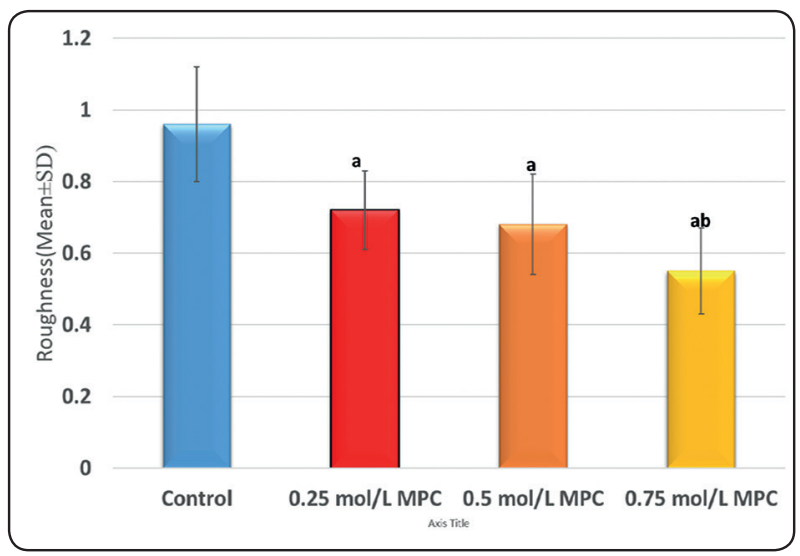

Fig. (2) Mean surface roughness (Ra) values for different groups.

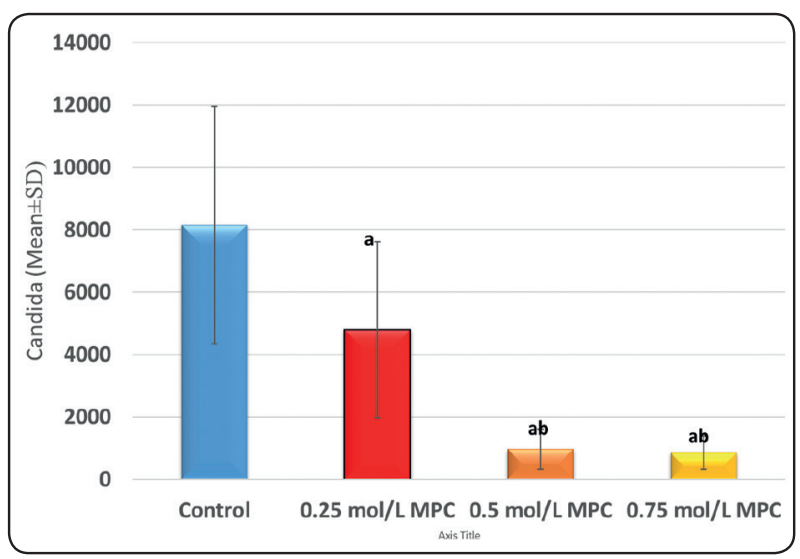

Fig. (3) Mean of adhered C. albicans numbers between different groups.

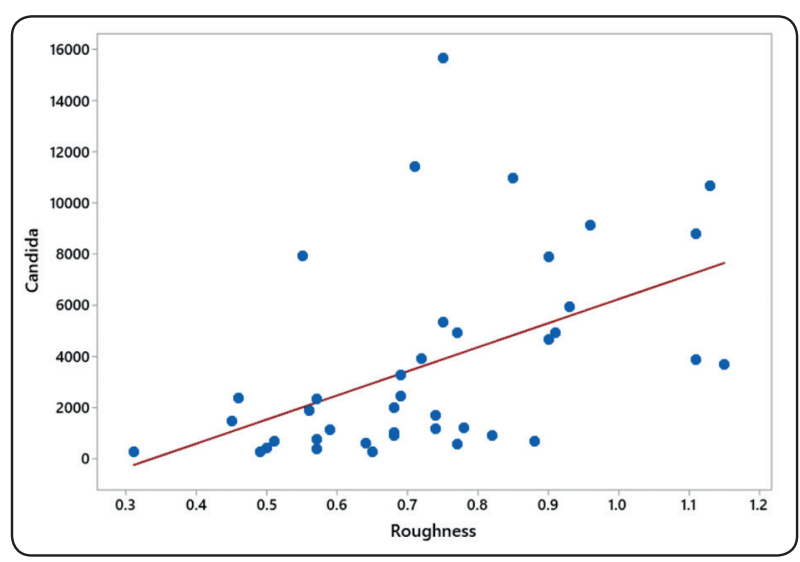

Fig. (4) Pearson correlations between SR and candida adhesion.

\section{DISCUSSION}

Polyamides are formed from, diamine with dibasic acid, $\mathrm{NH}_{2}-\left(\mathrm{CH}_{2}\right)_{6}-\mathrm{NH}_{2}, \quad \mathrm{CO}_{2} \mathrm{H}-\left(\mathrm{CH}_{2}\right)_{4}^{-}$ $\mathrm{COOH}$, by the condensation reactions between them. It is a crystalline polymer, and this structure accounts for good resistance to heat, ductility and protection from toxic effects of resin monomer and metal sensitivity, but it has many problems such as high surface roughness and bacterial contamination. $^{18}$ It has been reported that $\mathrm{C}$. albicans adhesion and other micro-organisms increased with increased hydrophobicity of the surfaces. ${ }^{19}$ Consequently, to reduce the attachment of microorganisms, the surface hydrophilicity would increase. ${ }^{20}$ Physical and chemical modification of denture base surface resulted in increasing the surface hydrophilicity, reducing energy of the surface, ${ }^{19,21,22}$ and adhesion of micro-organisms on acrylic denture base, with significant decrease in C. albicans attachment. ${ }^{23,24}$ Previous studies ${ }^{19,21}$, examined the influence of modification of acrylic resin surface with hydrophilic polymers, such as 2-hydroxyethyl methacrylate, 3-hydroxypropyl methacrylate and 2-trimethylammonium ethyl methacrylate chloride, they concluded that these coatings produced significantly lower C. albicans attachment to the acrylic resin and recommended that for prevention of denture stomatitis. Recently, 
2-methacryloyloxyethyl phosphoryl choline (MPC) polymer can be added to different restorative materials such as composite and also glass ionomer cements that used for banding to act as bacterialrepellent cement. ${ }^{16} \mathrm{MPC}$ is a methacrylate containing a phospholipid polar structure in the lateral chain which combines both hydrophilic heads and hydrophobic tails. ${ }^{13,25}$ As soon as water immersed of MPC, the phospholipids turned to a bilayer, the inner area of the bilayer directed towards the nonpolar tails, while the polar heads directed outward thus reacted with water. The protein-repelling polymer surface would reduce biofilm formation, protein adsorption and bacterial adhesion. ${ }^{26}$

Surface modification with MPC polymer was performed with numerous methods..$^{27,28}$ Coating of substrate surfaces, plasma treatment ${ }^{29}$ and graft polymerization ${ }^{14}$ are commonly used methods. It was found that, the surfaces that modified by MPC graft polymerization were relatively constant due to formation of chemical bonds between the polymer and denture base resin. ${ }^{11}$ By using this novel method, numerous monomers were efficiently fixed on solid elements, producing variation of efficient grafted particles. The concept of this method depending on creating a redox surface-initial system at the particle and the solution interface, subsequently, an excessive number of free radicals be formed onto solid particles surface, also the monomer easily starts to be polymerized on the particles producing well-organized function and improved strength..$^{30}$

Increased surface roughness results in microbial retaining and plaque accumulation, the rough surface contains irregularities such as voids and micro cracks, providing an increase microorganism retention, candida adhesion and protection from shear forces. ${ }^{31,32}$ This study found that surface roughness values significantly reduced for MPC coated surfaces resulting in smoother surfaces. This explained by the fact that these coating agents filled the microdefects on the resin surface creating smooth one. ${ }^{33}$ This is in agreement with other studies ${ }^{34-35}$ which reported the effect of coating agents on the reduction of surface roughness. Along with the results of the present study, previous studies, ${ }^{34,36}$ reported that denture base resin that coated with a light cured surface sealant attained less rough surface than uncoated one, afterward diminishing plaque adhesion and bacterial colonization. This is consistent with results stated for coating on PMMA denture base with Parylene ${ }^{35}$ and coating restorative CAD/CAM resin blocks with Optiglaze. ${ }^{37}$ Radford et al. ${ }^{38}$ reported that with an increase of the surface roughness from $2.5 \mu \mathrm{m}$ to $1.6 \mu \mathrm{m}$, there was corresponding increase of $C$. albicans adhesions on a resin base to 2.5 times. Previous research studying the effect of coating dental composite resins with an adhesive on its surface roughness, the adhesive was modified with various concentrations of MPC, a significant reduction in surface roughness values was noticed corresponding to the increase of MPC concentration. ${ }^{16}$ This is assured by the correlation results of our study, which demonstrate a significant positive correlation between SR and candida adhesion. In contrast to this result, a previous study $^{39}$ found a slight rise in surface roughness values parallel to the increase in MPC polymer ratios.

The result of our study revealed that there was statistically significant decrease of $C$. albicans adhesion with increased MPC concentrations. The reduction in $C$. albicans count was supposed to be associated with the rise in the hydrophilicity of the modified surfaces test groups and also, related to the significant decrease of surface roughness. This is in agreement with a former study ${ }^{40}$ which evaluated effects of coating PMMA with a film of nanocomposite silanated with $\mathrm{SiO}_{2}$ on its surface characteristics, in addition to adhesion of $C$. albicans, a reduction of Candida albicans count was noticed on the modified resin specimens without significant effect on the physical properties. 
The differences of the adhesion of $C$. albicans among examined groups were statistically significant, but there was no significant difference between the MPC modified groups with the concentrations of $(0.50 \mathrm{~mol} / \mathrm{L}$ and $0.75 \mathrm{~mol} / \mathrm{L})$. According to this result, coating the polyamide resin surface with MPC by graft polymerization method was determined to reduce $C$. albicans attachment significantly at whole tested concentrations of MPC in a comparison to the unmodified group. The lowest roughness value was recorded for 0.75 $\mathrm{mol} / \mathrm{L}$ MPC $(0.55 \pm 0.12)$, as it showed significantly decreased Ra compared to $0.50 \mathrm{~mol} / \mathrm{L}$. Therefore, polyamide surface modification with MPC in a monomer density of $0.75 \mathrm{~mol} / \mathrm{L}$ is suggested to be efficient, as it recorded decreased surface roughness and candida adhesion. While a previous study ${ }^{39}$ recommended the MPC modification with $0.5 \mathrm{~mol} /$ $\mathrm{L}$ concentration for coating acrylic denture base which recorded low candida adhesion with slight increase in surface roughness. This result can be explained by the difference in composition of both denture base materials. Additional studies needed to be made with other MPC concentrations assessing the mechanical and biological properties of the modified material. Also, bond strength, surface chemical composition and the interaction of MPC polymer on polyamide resin surface needed to be evaluated

\section{CONCLUSIONS}

1. Within the limitations of our study, alteration of the surface of polyamide denture base with MPC initiated an inhibitory influence on the adhesion of C.albicans.

2. Modification of the denture with MPC significantly decreased its surface roughness.

3. Modification with $0.75 \mathrm{~mol} / \mathrm{L} \mathrm{MPC}$ is recommended as it gives the best results

\section{REFERENCES}

1. Craig RG, Powers JM, et al. Restorative Dental Materials. 11th ed. St Louis: Mo, Mosby; 2002;636-689.

2. Weaver RE, Goebel WM. Reactions to acrylic resin dental prostheses. J Prosthet Dent. 1980;43:138-142.

3. Morgan TD, Wilson M. The effects of surface roughness and type of denture acrylic on biofilm formation by Streptococcus oral is in a constant depth film fermenter. J Appl Microbiol. 2001;91:47-53.

4. Abuzar MA, Bellur S, Duong N, et al. Evaluating surface roughness of a polyamide denture base material in comparison with poly (methyl methacrylate). J Oral Sci. 2010;52:577-581.

5. de Freitas Fernandes FS, Pereira-Cenci T, da Silva WJ, et al. Efficacy of denture cleansers on Candida spp. biofilm formed on polyamide and polymethyl methacrylate resins. J Prosthet Dent. 2011;105:51-58.

6. Salman M, Saleem S. Effect of different denture cleanser solutions on some mechanical and physical properties of nylon and acrylic denture base materials. J Baghdad College Dentistry 2011;23:19-24.

7. Kohli S, Bharita S. Polyamides in dentistry. Int J Scientific Study 2013;1(1):20-5.

8. Mai S. El-Din1, Amr M. Badr, Emad M Agamy, Gehan F Mohamed. Effect of two polishing techniques on surface roughness of three different denture base materials (an in vitro study). ADJALEXU.2018;43:34-40.

9. Akpan A, Morgan R. Oral candidiasis. Postgrad Med J. 2002;78:455-9.

10. Samaranayake LP, Keung Leung W, Jin L. Oral mucosal fungal infections. Periodontol 2000. 2009;49:39-59.

11. Takahashi N, Iwasa F, Inoue Y, Morisaki H, Ishihara K, Baba K. Evaluation of the durability and antiadhesive action of 2-methacryloyloxyethyl phosphorylcholine grafting on an acrylic resin denture base material. J Prosthet Dent. 2014;112:194-203.

12. Iwasaki Y, Aiba Y, Morimoto N, Nakabayashi N, Ishihara K. Semi-interpenetrating polymer networks composed of biocompatible phospholipid polymer and segmented polyurethane. J Biomed Mater Res. 2000;52:701-8

13. Ishihara K, Ueda T, Nakabayashi N. Preparation of phospholipid polymers and their properties as polymer hydrogel membranes. Polym J. 1990;22:355-60.

14. Tateishi T, Kyomoto M, Kakinoki S, Yamaoka T, Ishihara $\mathrm{K}$. Reduced platelets and bacteria adhesion on poly (ether ether ketone) by photoinduced and self-initiated graft polymerization of 2-methacryloyloxyethyl phosphorylcholine. J Biomed Mater Res A. 2014;102:1342-9 
15. Ishihara K, Oshida H, Endo Y, Watanabe A, Ueda T, Nakabayashi N. Effects of phospholipid adsorption on nonthrombogenicity of polymer with phospholipid polar group. J Biomed Mater Res. 1993;27:1309-14

16. Lee J. Inhibition of bacterial adhesion by poly (2-methacryloyloxyethyl phosphorylcholine) coating on dental composite resin. Seoul: Yonsei University; 2013.

17. Zhang N, Ma J, Melo MaS, Weir MD, Bai Y, HHK Xu. Protein-repellent and antibacterial dental composite to inhibit biofilms and caries. J Dent. 2015; 43:225-34.

18. Vojdani M, Giti R. Polyamide as a Denture Base Material: A Literature Review. J Dent (Shiraz) 2015,16:1-9.

19. Lazarin AA, Machado AL, Zamperini CA, et al: Effect of experimental photopolymerized coatings on the hydrophobicity of a denture base acrylic resin and on Candida albicans adhesion. Arch Oral Biol 2013;58:1-9.

20. Ali AA, Alharbi FA, Suresh CS: Effectiveness of coating acrylic resin dentures on preventing Candida adhesion. J Prosthodont 2013;22:445-450.

21. Izumida FE, Moffa EB, Vergani CE, Machado AL, Jorge $\mathrm{JH}$, Giampaolo ET. In vitro evaluation of adherence of Candida albicans, Candida glabrata, and Streptococcus mutans to an acrylic resin modified by experimental coatings. Biofouling. 2014;30:525-33.

22. Park SE, Periathamby AR, Loza JC. Effect of surfacecharged poly(methyl methacrylate) on the adhesion of Candida albicans. J Prosthodont. 2003;12:249-54.

23. Vukosavljevic D, Custodio W, Del Bel Cury AA, Siqueira WL. The effect of histatin 5, adsorbed on PMMA and hydroxyapatite, on Candida albicans colonization. Yeast. 2012;29:459-66.

24. Redding S, Bhatt B, Rawls HR, Siegel G, Scott K, LopezRibot J. Inhibition of Candida albicans biofilm formation on denture material. Oral Surg Oral Med Oral Pathol Oral Radiol Endod. 2009;107:669-72.

25. Mashaghi S, Jadidi T, Koenderink G, Mashaghi A. Lipid Nanotechnology. Int J Mol Sc. 2013; 14: 4242-4282

26. Lewis AL, Tolhurst LA, Stratford PW. Analysis of a phosphoryl choline-based polymer coating on a coronary stent pre-and post-implantation. Biomater 2002; 23: 1697-1706.

27. Sibarani J, Takai M, Ishihara K. Surface modification on microfluidic devices with 2-methacryloyloxyethyl phosphorylcholine polymers for reducing unfavorable protein adsorption. Colloids Surf B Biointerfaces. 2007;54:88-93.

28. Hirota K, Murakami K, Nemoto K, Miyake Y. Coating of a surface with 2-methacryloyloxyethyl phosphorylcholine (MPC) co-polymer significantly reduces retention of human pathogenic microorganisms. FEMS Microbiol Lett. 2005;248:37-45.
29. Huang X-D, Yao K, Zhang H, Huang X-J, Xu Z-K. Surface modification of silicone intraocular lens by 2-methacryloyloxyethyl phosphoryl-choline binding to reduce Staphylococcus epidermidis adherence. Clin Exp Ophthalmol. 2007;35:462-7.

30. Du R, Gao B, Men J, An F. Characteristics and advantages of surface-initiated graft-polymerization as a way of "grafting from" method for graft-polymerization of functional monomers on solid particle. eurpolymj.2020;127:109479.

31. Nevzatoglu EU, Ozcan M, Kulak-Ozkan Y, Kadir T. Adherence of Candida albicans to denture base acrylics and silicone-based resilient liner materials with different surface finishes. Clin Oral Investig. 2007;11:231-6.

32. Kawai K, Uranom Ebisus. Effect of surface roughness of porcelain on adhesion of bacteria arc their synthesis glucan. J Prosthet Dent. 2000;83:664-7.

33. Sahin O, Koroglu A, Dede DO, et al: Effect of surface sealant agents on the surface roughness and color stability of denture base materials. J Prosthet Dent 2016;116:610-616.

34. Dayrell A, Takahashi J, Valverde G, et al: Effect of sealer coating on mechanical and physical properties of permanent soft lining materials. Gerodontology 2012;29:401407.

35. Bourlidi S, Qureshi J, Soo S, et al: Effect of different initial finishes and Parylene coating thickness on the surface properties of coated PMMA. J Prosthet Dent 2016;115:363-370.

36. Sesma N, Lagana DC, Morimoto S, et al: Effect of denture surface glazing on denture plague formation. Braz Dent $\mathrm{J}$ 2005;15:55-58.

37. Tekc, e N, Fidan $S$, Tuncer $S$, et al: The effect of glazing and aging on the surface properties of CAD/CAM resin blocks. J Adv Prosthodont 2018;10:50-57.

38. Radford DR, Sweet SP, Challacombe SJ, Walter JD. Adherence of Candida albicans to denture-base materials with different surface finishes. J Dent 1998; 26:577-83.

39. Türkcan I, Nalbant D, Bat E, Akca G. Examination of 2-methacryloyloxyethyl phosphorylcholine polymer coated acrylic resin denture base material: surface characteristics and Candida albicans adhesion. J Sci Mater Med. 2018, 29:107

40. Yodmongkol S, Chantarachindawong R, Thaweboon S, Thaweboon B, Amornsakchai T, Srikhirin T. The effects of silane- $\mathrm{SiO}_{2}$ nanocomposite films on Candida albicans adhesion and the surface and physical properties of acrylic resin denture base material. J Prosthet Dent. 2014; 112:1530-8. 